\title{
Analysis of the MILES cohort reveals determinants of disease progression and treatment response in lymphangioleiomyomatosis
}

\author{
Nishant Gupta ${ }^{1}$, Hye-Seung Lee ${ }^{2}$, Lisa R. Young ${ }^{3}$, Charlie Strange ${ }^{4}$, Joel Moss ${ }^{5}$, \\ Lianne G. Singer ${ }^{6}$, Koh Nakata ${ }^{7}$, Alan F. Barker ${ }^{8}$, Jeffrey T. Chapman ${ }^{9}$, \\ Mark L. Brantly ${ }^{10}$, James M. Stocks ${ }^{11}$, Kevin K. Brown ${ }^{12}$, Joseph P. Lynch 3rd ${ }^{13}$, \\ Hilary J. Goldberg ${ }^{14}$, Gregory P. Downey ${ }^{12}$, Angelo M. Taveira-DaSilva ${ }^{5}$, \\ Jeffrey P. Krischer ${ }^{2}$, Kenneth Setchell ${ }^{15}$, Bruce C. Trapnell ${ }^{1,15}$, Yoshikazu Inoue ${ }^{16}$ \\ and Francis X. McCormack ${ }^{1}$ for the NIH Rare Lung Disease Consortium
}

@ERSpublications

Menopausal status and serum vascular endothelial growth factor-D levels are clinically useful variables that should be taken into consideration when making therapeutic decisions and designing clinical trials for patients with lymphangioleiomyomatosis http://ow.ly/ijGB30nrNCB

Cite this article as: Gupta N, Lee H-S, Young LR, et al. Analysis of the MILES cohort reveals determinants of disease progression and treatment response in lymphangioleiomyomatosis. Eur Respir J 2019; 53: 1802066 [https://doi.org/10.1183/13993003.02066-2018].

\section{ABSTRACT}

Introduction: The Multicenter International Lymphangioleiomyomatosis (LAM) Efficacy of Sirolimus (MILES) trial revealed that sirolimus stabilised lung function in patients with moderately severe LAM. The purpose of this study was to further examine the MILES cohort for the effects of racial, demographic, clinical and physiological patient characteristics on disease progression and treatment response in LAM. Methods: MILES subjects were stratified on the basis of menopausal status (pre-menopausal/postmenopausal), race (Asian/Caucasian), bronchodilator responsiveness (present/absent), initial forced expiratory volume in $1 \mathrm{~s}$ (FEV1; $51-70 \%$ versus $\leqslant 50 \%$ predicted) and tuberous sclerosis complex (TSC) association (yes/ no). A linear mixed effects model was used to compare slope differences, and nonparametric tests were used to compare medians and proportions between treatment groups in each stratum.

Results: In the MILES placebo group, pre-menopausal patients declined 5-fold faster than post-menopausal patients (mean \pm SE FEV1 slope $-17 \pm 3$ versus $-3 \pm 3 \mathrm{~mL} \cdot \mathrm{month}^{-1} ; \mathrm{p}=0.003$ ). Upon treatment with sirolimus, both the pre-menopausal $\left(-17 \pm 3\right.$ versus $\left.-1 \pm 2 \mathrm{~mL} \cdot \mathrm{month}^{-1} ; \mathrm{p}<0.0001\right)$ and post-menopausal patients $(-3 \pm 3$ versus $6 \pm 3 \mathrm{~mL} \cdot$ month $\left.^{-1} ; \mathrm{p}=0.04\right)$ exhibited a beneficial response in mean $\pm \mathrm{SE} F E V 1$ slope compared with the placebo group. Race, LAM subtype, bronchodilator responsiveness or baseline FEV1 did not impact the rate of disease progression in the placebo group or treatment response in the sirolimus group. Menopausal status and race had differential effects on the adverse event profile of sirolimus. Baseline serum vascular endothelial growth factor (VEGF)-D $>600 \mathrm{pg} \cdot \mathrm{mL}^{-1}$ identified subgroups of patients who were more likely to decline on placebo and respond to treatment with sirolimus.

Conclusions: In LAM patients, treatment with sirolimus is beneficial regardless of menopausal status, race, bronchodilator responsiveness, baseline FEV1 or TSC association. Serum VEGF-D and menopausal status can help inform therapeutic decisions.

This article has supplementary material available from erj.ersjournals.com

Received: Oct 292018 | Accepted after revision: Jan 192019

The content of this work is not subject to copyright. Design and branding are copyright @ERS 2019. 


\section{Introduction}

Lymphangioleiomyomatosis (LAM) is a rare cystic lung disease that produces respiratory failure in females. LAM can occur sporadically (S-LAM) or in association with the heritable disease, tuberous sclerosis complex (TSC-LAM) [1]. In both TSC-LAM and S-LAM, loss-of-function mutations in TSC genes result in constitutive activation of the mechanistic target of rapamycin (mTOR) signalling pathway, leading to inappropriate LAM cell growth, invasion, migration, lymphangiogenesis and destructive tissue remodelling [2]. The average age at diagnosis of LAM is $~ 35$ years [1] and the typical rate of decline in forced expiratory volume in $1 \mathrm{~s}\left(\mathrm{FEV}_{1}\right)$ has been variably reported as $75-135 \mathrm{~mL}$-year ${ }^{-1}[3,4]$. In a recent randomised controlled trial, the Multicenter International LAM Efficacy of Sirolimus (MILES) trial, the mTOR inhibitor sirolimus was shown to stabilise lung function and to improve some measures of quality of life in patients with LAM [4]. Adverse events were frequent and consistent with those known to be associated with mTOR inhibitor use, but serious adverse events were balanced between the placebo and sirolimus groups [4].

Various retrospective reports have identified factors that may impact the natural history of LAM. For instance, post-menopausal females with LAM have a lower rate of decline in FEV1 compared with pre-menopausal females $[3,5,6]$. Race has been mentioned as a possible disease-modifying factor, with reduced rates of decline in FEV1 reported among Asian females with LAM compared with Caucasian LAM patients [6,7]. The presence of bronchodilator responsiveness on spirometry has been linked to faster rate of decline in FEV1 [5, 8]. Patients with TSC-LAM are believed to have milder and less progressive disease compared with S-LAM $[9,10]$. The diagnostic and predictive value of serum vascular endothelial growth factor (VEGF)-D is well established [11-13], but there exist conflicting reports with regard to the prognostic value of the biomarker [5]. The purpose of the current study was to exploit the prospective design of the MILES trial to investigate the impact of key demographic and clinical features on the natural history of lung function decline and response to treatment with sirolimus. Some of the results from this analysis have been previously published in abstract form $[14,15]$.

\section{Methods}

\section{Background and study population}

Our study population included the participants enrolled in the MILES trial (ClinicalTrials.gov identifier NCT00414648), a randomised, controlled trial where patients with a confirmed diagnosis of LAM and $\mathrm{FEV}_{1} \leqslant 70 \%$ predicted were randomly assigned in a double-blind fashion to receive sirolimus or placebo for 1 year, followed by 1 year of observation. The initial dose of sirolimus was $2 \mathrm{mg}$ daily, which was dose-adjusted in a blinded fashion to maintain a blood trough level of 5-15 ng.mL $\mathrm{m}^{-1}$. Pulmonary function tests (PFTs), serum VEGF-D levels and patient-reported outcomes were gathered serially. Data from the second trial year, in which patients were observed off therapy, were not included in the current analysis.

\section{Procedures}

PFT methodology from the MILES trial has been previously reported [4]. All PFTs were performed in accordance with the American Thoracic Society (ATS)/European Respiratory Society (ERS) criteria [16-18], with real-time feedback to maintain quality of spirometry through the trial. The presence or absence of bronchodilator responsiveness was determined in all trial subjects at the baseline visit as per the ATS/ERS criteria $[16,19]$. Spirometry was performed at baseline and every 3 months in the first year.

Menopausal status, either natural (defined by the absence of menstrual flow for a period of at least 12 months) or surgical (defined as surgical removal of the uterus with/without removal of the ovaries), was determined based on history at the enrolment visit. Serum VEGF-D concentrations were measured at baseline, and 6 and 12 months. VEGF-D testing was done in a College of American Pathologists (CAP)/

Affiliations: ${ }^{1}$ University of Cincinnati, Cincinnati, OH, USA. ${ }^{2}$ University of South Florida, Tampa, FL, USA. ${ }^{3}$ Vanderbilt University Medical Center, Nashville, TN, USA. ${ }^{4}$ Medical University of South Carolina, Charleston, SC, USA. ${ }^{5}$ National Heart, Lung, and Blood Institute, National Institutes of Health, Bethesda, MD, USA. ${ }^{6}$ University of Toronto, Toronto, ON, Canada. ${ }^{7}$ Niigata University Medical and Dental Hospital, Niigata, Japan. ${ }^{8}$ Oregon Health and Science University, Portland, OR, USA. ${ }^{9}$ Cleveland Clinic, Abu Dhabi, United Arab Emirates. ${ }^{10}$ University of Florida, Gainesville, FL, USA. ${ }^{11}$ University of Texas Health Sciences Center, Tyler, TX, USA. ${ }^{12}$ National Jewish Health and the University of Colorado, Denver, CO, USA. ${ }^{13}$ University of California, Los Angeles, CA, USA. ${ }^{14}$ Harvard Medical School, Boston, MA, USA. ${ }^{15}$ Cincinnati Children's Hospital Medical Center, Cincinnati, OH, USA. ${ }^{16}$ National Hospital Organization Kinki-Chuo Chest Medical Center, Osaka, Japan.

Correspondence: Nishant Gupta, Division of Pulmonary, Critical Care, and Sleep Medicine, University of Cincinnati, MSB Room 6053, ML 0564, 231 Albert Sabin Way, Cincinnati, OH 45267-0564, USA.

E-mail: Nishant.guptaduc.edu 
Clinical Laboratory Improvement Amendments (CLIA)-accredited laboratory, by technicians masked to treatment assignment and clinical data. A modified form of the Quantikine Human VEGF-D Immunoassay (R\&D Systems, Minneapolis, MN, USA) was used for the measurements.

\section{Statistical analysis}

For this study, the various analyses were stratified according to the condition of interest. A linear mixed effects model was used to compare slope differences, and nonparametric tests were used to compare medians and proportions between treatment groups in each stratum. In the linear mixed effects model analysis, we used the Kenward-Roger correction to adjust the degree of freedom to improve performance when data were missing [20]. For categorical outcomes, the data were compared with the use of Fisher's exact test. For continuous variables, the medians were compared with the use of the Wilcoxon rank-sum test. The $95 \%$ confidence interval for group differences was obtained from the mean estimates. With regard to adverse events, the mean number of events per subject is reported in each group. In order to ascertain significance level of the difference between the various subgroups of interest, we compared the median subject-specific proportions of adverse events by using the Wilcoxon rank-sum test. We deemed p-values $<0.05$ to be significant. All reported p-values are two-sided and unadjusted for multiple testing. All statistical analyses were performed using SAS version 9.4 (SAS Institute, Cary, NC, USA).

\section{Results}

In the MILES trial, a total of 89 patients were enrolled across 10 sites, including seven in the USA, two in Japan and one in Canada; 43 patients were randomised to the placebo arm and 46 to the sirolimus arm. At the end of the first year, there were 34 remaining patients in the placebo arm and 41 patients in the sirolimus arm. The mean \pm SD age of enrolled patients was $45.4 \pm 10.6$ years. 30 (34\%) patients in the MILES cohort were post-menopausal, including 16 out of 43 (37\%) patients in the placebo group and 14 out of 46 (30\%) patients in the sirolimus group. 59 patients (66\%) in the MILES cohort were pre-menopausal, including 27 out of $59(46 \%)$ patients in the placebo group and 32 out of 59 (54\%) patients in the sirolimus group. At the end of the first year, in the post-menopausal group, there were 13 remaining patients in the placebo arm and 11 patients in the sirolimus arm. Similarly, in the pre-menopausal group, at the end of the first year, there were 21 remaining patients in the placebo arm and 30 patients in the sirolimus arm. There were 27 (30\%) Asian patients: 12 in the placebo arm and 15 in the sirolimus arm. At the end of the first year, there were 10 remaining patients in the placebo arm and 13 in the sirolimus arm. There were 62 (70\%) Caucasian patients: 31 each in the placebo and sirolimus arms. At the end of the first year, there were 24 remaining patients in the placebo arm and 28 in the sirolimus arm.

In the overall MILES cohort, the baseline lung function and VEGF-D levels were similar in the placebo and treatment arms [4]. In the current analysis, the pre-menopausal/post-menopausal and Asian/Caucasian subgroups of the placebo and sirolimus groups remained similarly matched (supplementary tables E1 and E2), except post-menopausal patients in the placebo arm had a higher baseline diffusion capacity of the lung for carbon monoxide (DLCO) compared with the sirolimus arm $\left(12.09\right.$ versus $9.42 \mathrm{~mL} \cdot \mathrm{mmHg}^{-1} \cdot \mathrm{min}^{-1}$; $\mathrm{p}=0.05$ ) and the baseline FEV1 was higher in the sirolimus subgroup than the placebo subgroup in the Asian cohort (1.43 versus $1.11 \mathrm{~L} ; \mathrm{p}=0.05)$.

\section{Menopause}

Pulmonary function tests

In the placebo group, pre-menopausal subjects exhibited a significantly faster mean \pm SE rate of decline in FEV 1 compared with the post-menopausal subjects $\left(-17 \pm 3\right.$ versus $\left.-3 \pm 3 \mathrm{~mL} \cdot \mathrm{month}^{-1} ; \mathrm{p}=0.003\right)$. Similar to FEV1, pre-menopausal females in the placebo group had a faster mean \pm SE rate of decline in forced vital capacity $(\mathrm{FVC})$ compared with the post-menopausal females $\left(-17 \pm 4\right.$ versus $-3 \pm 4 \mathrm{~mL} \cdot \mathrm{month}^{-1}$; $\left.\mathrm{p}=0.01\right)$. In the pre-menopausal placebo group, the mean \pm SE FEV1 slope $\left(-17 \pm 3 \mathrm{~mL} \cdot \mathrm{month}^{-1}\right)$ was significantly less than zero $(\mathrm{p} \leqslant 0.0001)$, a finding that was consistent with declining lung function. The mean $\pm \mathrm{SE} F E V 1$ slope in the sirolimus group $\left(-1 \pm 2 \mathrm{~mL} \cdot \mathrm{month}^{-1}\right)$ was not significantly different than zero $(\mathrm{p}=0.66)$; this was indicative of the stabilisation of lung function during treatment. In the post-menopausal placebo group, the mean \pm SE FEV1 slope $\left(-3 \pm 3 \mathrm{~mL} \cdot \mathrm{month}^{-1}\right)$ was not significantly different than zero $(\mathrm{p}=0.25)$, a finding that was consistent with stable lung function. The mean \pm SE FEV1 slope in the post-menopausal sirolimus group $\left(6 \pm 3 \mathrm{~mL} \cdot \mathrm{month}^{-1}\right)$, although not statistically different than zero, had a trend towards significance $(\mathrm{p}=0.08)$, indicating potential improvement of lung function during treatment. When compared with placebo, sirolimus treatment resulted in a beneficial response in FEV1 and FVC slope in both the pre-menopausal and post-menopausal groups (table 1, and figures 1a and 2a). The rate of decline of DLCO was not different in the placebo and sirolimus groups in the overall MILES cohort or in the pre-menopausal patients $\left(-0.07\right.$ versus $\left.-0.02 \mathrm{~mL} \cdot \mathrm{mmHg}^{-1} \cdot \mathrm{min}^{-1} \cdot \mathrm{month}^{-1} ; \mathrm{p}=0.4\right)$. However, in the 
TABLE 1 Change from baseline to 12 months in outcomes after dividing the MILES cohort into pre- and post-menopausal subgroups

Pre-menopausal

Placebo Sirolimus Between-group p-value
difference $95 \% \mathrm{Cl}$
Post-menopausal

Placebo Sirolimus Between-group p-value difference $95 \% \mathrm{Cl}$

\begin{tabular}{|c|c|c|c|c|c|c|c|c|}
\hline Subjects & 27 & 32 & & & 16 & 14 & & \\
\hline Rate of decline of FEV $1 \mathrm{~mL} \cdot \mathrm{month}^{-1}$ & $-17 \pm 3$ & $-1 \pm 2$ & $9.38-22.28$ & $<0.0001$ & $-3 \pm 3$ & $6 \pm 3$ & $0.73-17.45$ & 0.04 \\
\hline Rate of decline of FVC $\mathrm{mL} \cdot \mathrm{month}^{-1}$ & $-17 \pm 4$ & $6 \pm 4$ & $11.93-33.21$ & 0.0001 & $-3 \pm 4$ & $16 \pm 6$ & $4.52-33.28$ & 0.03 \\
\hline $\begin{array}{l}\text { Rate of decline of } D \text { Lco } \\
\qquad \mathrm{mL} \cdot \mathrm{mmHg}^{-1} \cdot \mathrm{min}^{-1} \cdot \mathrm{month}^{-1}\end{array}$ & $-0.07 \pm 0.04$ & $-0.02 \pm 0.03$ & $-0.048-0.148$ & 0.4 & $-0.04 \pm 0.02$ & $0.03 \pm 0.03$ & $-0.00-0.14$ & 0.04 \\
\hline Serum VEGF-D slope $\mathrm{pg} \cdot \mathrm{mL}^{-1} \cdot \mathrm{month}^{-1}$ & $-15 \pm 20$ & $-99 \pm 18$ & $31.24-136.56$ & 0.003 & $22 \pm 27$ & $-54 \pm 31$ & $-3.93-156.93$ & 0.07 \\
\hline Change in 6MWD $\mathrm{m}$ & $31 \pm 10$ & $24 \pm 10$ & $-21.73-35.35$ & 0.63 & $18 \pm 16$ & $23 \pm 21$ & $-46.72-55.56$ & 0.69 \\
\hline Change in VAS & $-2.8 \pm 3.8$ & $7 \pm 3.2$ & $0.04-19.54$ & 0.07 & $-1.5 \pm 3.4$ & $3.7 \pm 4.6$ & $-6.04-16.58$ & 0.55 \\
\hline Change in FPI & $-0.04 \pm 0.05$ & $0.10 \pm 0.07$ & $-0.03-0.31$ & 0.26 & $-0.07 \pm 0.06$ & $0.10 \pm 0.11$ & $-0.08-0.42$ & 0.14 \\
\hline
\end{tabular}

Data are presented as $\mathrm{n}$ or mean \pm SE, unless otherwise stated. FEV1: forced expiratory volume in $1 \mathrm{~s}$; FVC: forced vital capacity; DLco: diffusion capacity of the lung for carbon monoxide; VEGF: vascular endothelial growth factor; 6MWD: 6-min walk distance; VAS: visual analogue scale; $\mathrm{FPI}$ : functional performance inventory.

post-menopausal patients, there was a significant between-group difference in DLCO slope, favouring the sirolimus group $\left(-0.04\right.$ versus $\left.0.03 \mathrm{~mL} \cdot \mathrm{mmHg}^{-1} \cdot \mathrm{min}^{-1} \cdot \mathrm{month}^{-1} ; \mathrm{p}=0.04\right)$.

\section{VEGF-D response}

The mean \pm SE difference between placebo and sirolimus groups in VEGF-D slope was statistically significant in the pre-menopausal patients $\left(-15 \pm 20\right.$ versus $\left.-99 \pm 18 \mathrm{pg} \cdot \mathrm{mL}^{-1} \cdot \mathrm{month}^{-1} ; \mathrm{p}=0.003\right)$ and had a trend towards significance in the post-menopausal patients $\left(22 \pm 27\right.$ versus $-54 \pm 31 \mathrm{pg} \cdot \mathrm{mL}^{-1} \cdot \mathrm{month}^{-1}$; $\mathrm{p}=0.07$ ) (table 1 and figure $3 \mathrm{a}$ ).

\section{Adverse events}

Pre-menopausal patients who were treated with sirolimus had more dermatological adverse events (3.1 versus 1.7 events per subject; $\mathrm{p}=0.006$ ) during the 12 -month treatment period than pre-menopausal placebo patients. However, the sirolimus-treated post-menopausal patients had more gastrointestinal adverse events than the post-menopausal placebo patients (6.2 versus 3.2 events per subject; $\mathrm{p}=0.015)$.

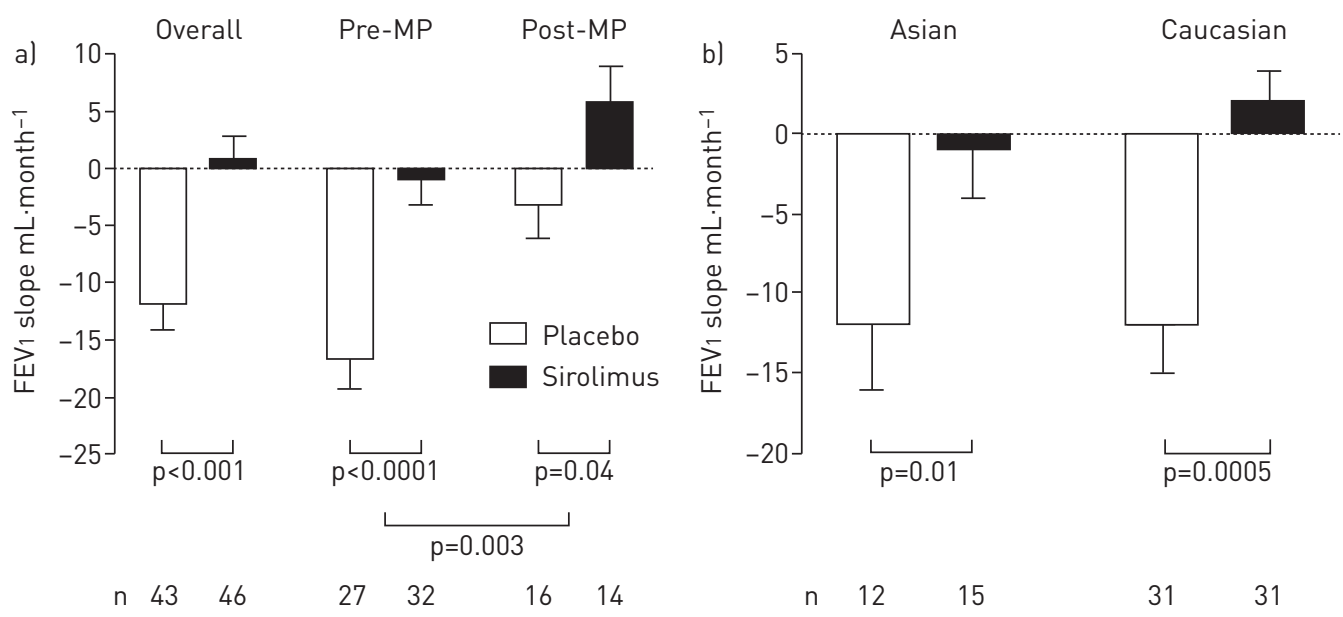

FIGURE 1 Effect of a) menopause and b) race on forced expiratory volume in $1 \mathrm{~s}$ (FEV 1 ) response. Pre-MP: pre-menopausal; post-MP: post-menopausal. a) Mean \pm SE FEV1 response by menopausal status. In the overall cohort, sirolimus stabilised FEV1 decline. In the placebo group, pre-MP females exhibited a significantly faster rate of decline of FEV1 compared with post-MP females. Both the pre-MP and post-MP females exhibited a beneficial response to treatment with sirolimus, with pre-MP females experiencing a slowing in the rate of decline and post-MP females trending towards improvement. b) Mean \pm SE FEV 1 response by race. In the overall cohort, sirolimus stabilised FEV 1 decline. There was no difference in the rate of decline of FEV 1 in the Asian and Caucasian patients enrolled in MILES, and both subgroups responded to treatment with sirolimus. 


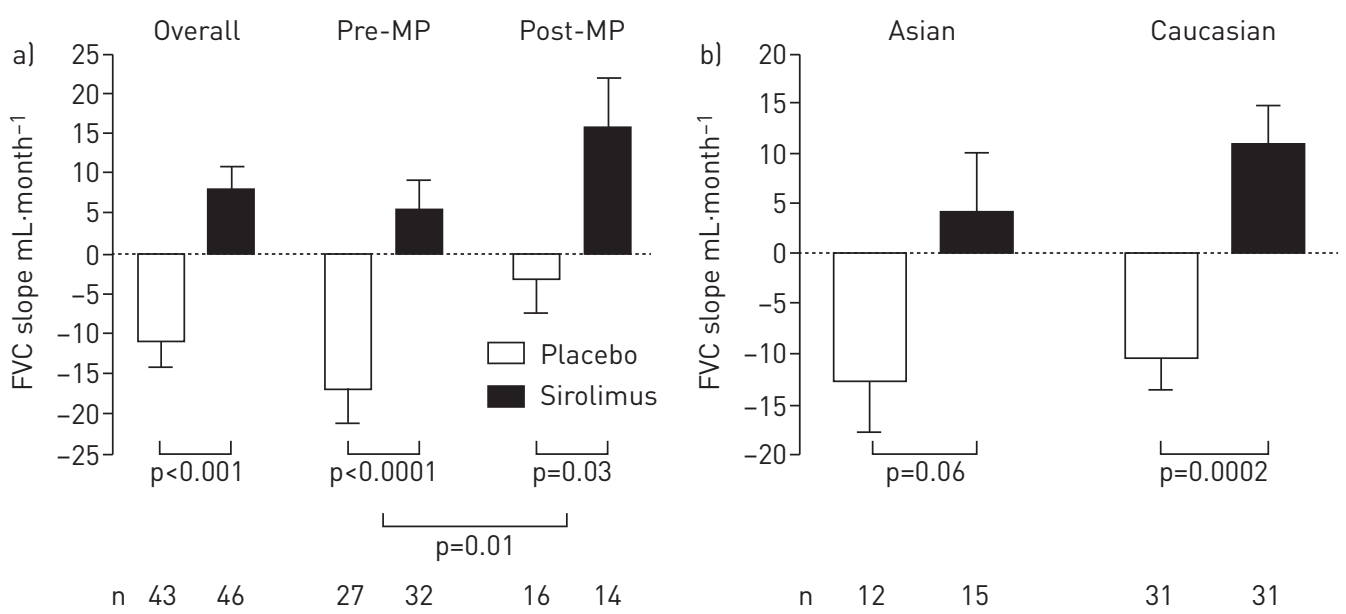

FIGURE 2 Effect of a) menopause and b) race on forced vital capacity (FVC) response. Pre-MP:

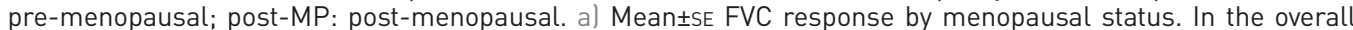
cohort, sirolimus stabilised FVC decline. In the placebo group, pre-MP females had a significantly faster rate of decline in FVC compared with post-MP females. Both the pre-MP and post-MP females had a beneficial response to treatment with sirolimus. b) Mean \pm SE FVC response by race. The rate of decline in FVC was similar in the placebo groups of both the Asian and Caucasian patients. The difference between placebo and sirolimus groups in FVC slope was statistically significant in the Caucasian patients, and trended towards significance in the Asian patients.

Comparing the sirolimus groups between pre- and post-menopausal subjects, there were no differences in the frequency or type of adverse events or in the frequency of serious adverse events.

Race

Pulmonary function tests

After dividing the MILES cohort on the basis of race, the rate of decline in lung function (FEV1, FVC and DLCO) was similar in the Asian and Caucasian placebo groups (table 2). Both racial subgroups had a beneficial response in their FEV1 and FVC slopes following treatment with sirolimus (table 2, and figures $1 \mathrm{~b}$ and $2 \mathrm{~b}$ ). There was no significant change in the rate of decline of DLCO after treatment with sirolimus in either the Asian and Caucasian cohorts (table 2).

\section{VEGF-D response}

The mean \pm SE serum VEGF-D levels declined significantly following treatment with sirolimus compared with placebo in both the Asian $\left(-17 \pm 27\right.$ versus $\left.-102 \pm 24 \mathrm{pg} \cdot \mathrm{mL}^{-1} \cdot \mathrm{month}^{-1} ; \mathrm{p}=0.03\right)$ and Caucasian $(3 \pm 22$ versus $-79 \pm 22 \mathrm{pg} \cdot \mathrm{mL}^{-1} \cdot \mathrm{month}^{-1} ; \mathrm{p}=0.01$ ) patients (table 2 and figure $3 \mathrm{~b}$ ).
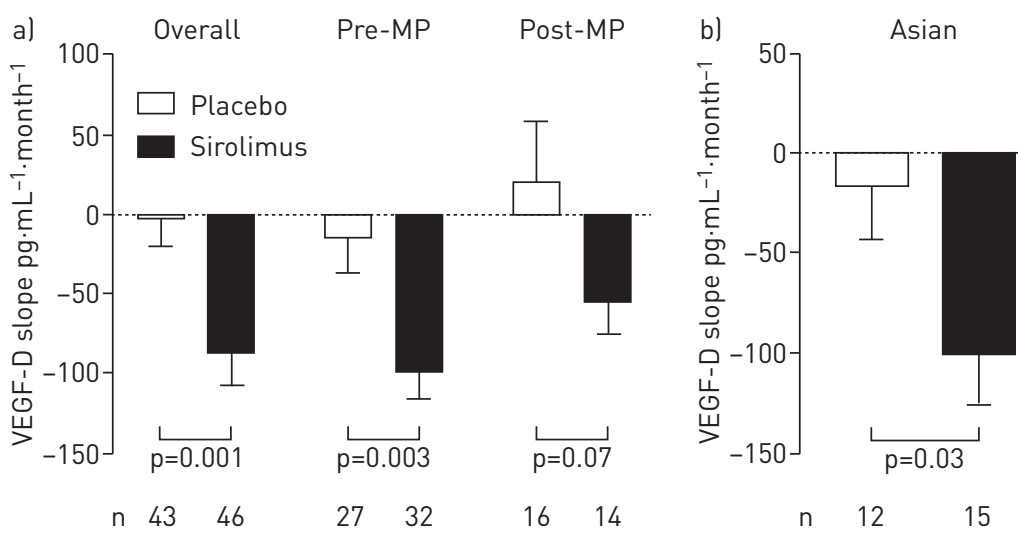

Caucasian

FIGURE 3 Effect of a) menopause and b) race on serum vascular endothelial growth factor (VEGF)-D response. Pre-MP: pre-menopausal; post-MP: post-menopausal. a) Mean \pm SE change in serum VEGF-D levels among pre-MP and post-MP patients. In the overall cohort, serum VEGF-D levels were stable over time in the placebo group and declined in the sirolimus group. This was also true in the pre-MP patients, but the between-group difference in post-MP patients did not achieve significance, likely due to the small patient numbers. b) Mean \pm SE change in serum VEGF-D levels among Asian and Caucasian patients. Both the Asian and Caucasian subjects exhibited a significant decline in serum VEGF-D levels following treatment with sirolimus. 
TABLE 2 Change from baseline to 12 months in outcomes after dividing the MILES cohort on the basis of race into Asian and Caucasian subgroups

\begin{tabular}{|c|c|c|c|c|c|c|c|c|}
\hline & \multicolumn{4}{|c|}{ Asian } & \multicolumn{4}{|c|}{ Caucasian } \\
\hline & Placebo & Sirolimus & $\begin{array}{l}\text { Between-group } \\
\text { difference } 95 \% \mathrm{Cl}\end{array}$ & p-value & Placebo & Sirolimus & $\begin{array}{l}\text { Between-group } \\
\text { difference } 95 \% \mathrm{Cl}\end{array}$ & p-value \\
\hline Rate of decline of FEV 1 mL-month ${ }^{-1}$ & $-12 \pm 3$ & $-1 \pm 3$ & $3.16-19.22$ & 0.01 & $-12 \pm 3$ & $2 \pm 3$ & $6.61-21.39$ & 0.0005 \\
\hline Rate of decline of $\mathrm{FVC} \mathrm{mL} \cdot \mathrm{month}^{-1}$ & $-13 \pm 5$ & $4 \pm 6$ & $1.59-32.21$ & 0.06 & $-11 \pm 3$ & $11 \pm 4$ & $11.6-31.2$ & 0.0002 \\
\hline $\begin{array}{l}\text { Rate of decline of } D \text { Lco } \\
\quad \mathrm{mL} \cdot \mathrm{mmHg}^{-1} \cdot \mathrm{min}^{-1} \cdot \mathrm{month}^{-1}\end{array}$ & $-0.07 \pm 0.04$ & $-0.04 \pm 0.03$ & $-0.07-0.13$ & 0.47 & $-0.05 \pm 0.04$ & $0.01 \pm 0.03$ & $-0.04-0.16$ & 0.26 \\
\hline Change in VAS & $-1.7 \pm 3.1$ & $5.4 \pm 3.2$ & $-1.6-15.8$ & 0.18 & $-3.8 \pm 5.2$ & $7.3 \pm 4.8$ & $-2.8-25.0$ & 0.20 \\
\hline Change in FPI & $-0.01 \pm 0.05$ & $0.02 \pm 0.05$ & $-0.1-0.2$ & 0.82 & $-0.14 \pm 0.06$ & $0.24 \pm 0.13$ & $0.1-0.7$ & 0.02 \\
\hline
\end{tabular}

Data are presented as $\mathrm{n}$ or mean $\pm \mathrm{SE}$, unless otherwise stated. $\mathrm{FEV} 1$ : forced expiratory volume in $1 \mathrm{~s}$; FVC: forced vital capacity; DLCO: diffusion capacity of the lung for carbon monoxide; VEGF: vascular endothelial growth factor; 6MWD: 6-min walk distance; VAS: visual analogue scale; FPI: functional performance inventory.

\section{Adverse events}

In the race-stratified analysis, dermatological adverse events were more common in the sirolimus group than the placebo group in Asian patients (3.4 versus 1.2 events per subject; $\mathrm{p}=0.03$ ), but not in Caucasian patients. The frequency of serious adverse events did not differ between Asian and Caucasian patients. Comparing the sirolimus groups between Asian and Caucasian patients, there were no differences in the frequency or type of adverse events.

\section{Tuberous sclerosis complex}

There were four TSC-LAM patients each in the placebo and sirolimus subgroups. There was no difference in the rate of decline of FEV1 in the placebo group, or the magnitude of FEV1 response in the treatment group, after dividing the MILES cohort into TSC-LAM and S-LAM subgroups (supplementary tables E3E5, and supplementary figure E1).

\section{Bronchodilator responsiveness}

Bronchodilator responsiveness was demonstrated at baseline in a total of 27 subjects (30.3\%) enrolled in the MILES trial. The rate of decline in FEV1 in the placebo group was similar regardless of the presence or absence of bronchodilator responsiveness $\left(-11.9\right.$ versus $\left.-11.8 \mathrm{~mL} \cdot \mathrm{month}^{-1} ; \mathrm{p}=0.98\right)$. Both subgroups exhibited a significant beneficial response to treatment with sirolimus (supplementary tables E6 and E7, and supplementary figure E2).

\section{Baseline FEV1}

The MILES cohort was divided on the basis of baseline FEV1 into two groups: $51-70 \%$ and $\leqslant 50 \%$ predicted. The rate of decline in FEV1 in the placebo group was similar regardless of the baseline FEV1. Both subgroups exhibited a significant beneficial response to treatment with sirolimus (supplementary tables E8 and E9, and supplementary figure E3).

\section{Serum VEGF-D as a predictor of disease progression and treatment response}

In a previous post hoc analysis of the MILES trial, each 1-log increase in baseline VEGF-D was shown to be associated with a $134 \mathrm{~mL}$ between-group difference in baseline to 12-month mean change in FEV1 [13]. In the current analysis, we examined the utility of a VEGF-D cut-off as a biomarker of progression and treatment response in the MILES subjects. At a VEGF-D serum level $>600 \mathrm{pg} \cdot \mathrm{mL}^{-1}$, patients in the placebo group were more likely to progress rapidly and to respond to treatment, whereas progression and treatment response were less robust in the group of patients with a serum VEGF-D level $\leqslant 600 \mathrm{pg} \cdot \mathrm{mL}^{-1}$ (figure $4 \mathrm{a}$ ). When percentage baseline to 12-month change in VEGF-D was plotted as a function of percentage baseline to 12 -month change in FEV1 in the placebo and sirolimus groups, the placebo group remained normally distributed around the origin, whereas the sirolimus group migrated towards the reduced VEGF-D/increased FEV1 quadrant (figure 5). 




Placebo-VEGF-D $\leqslant 600 \mathrm{pg} \cdot \mathrm{mL}^{-1}$

Placebo-VEGF-D >600 pg. $\mathrm{mL}^{-1}$ $\square$ Sirolimus-VEGF-D $\leqslant 600 \mathrm{pg} \cdot \mathrm{mL}^{-1}$

Sirolimus-VEGF-D $>600 \mathrm{pg} \cdot \mathrm{mL}^{-1}$

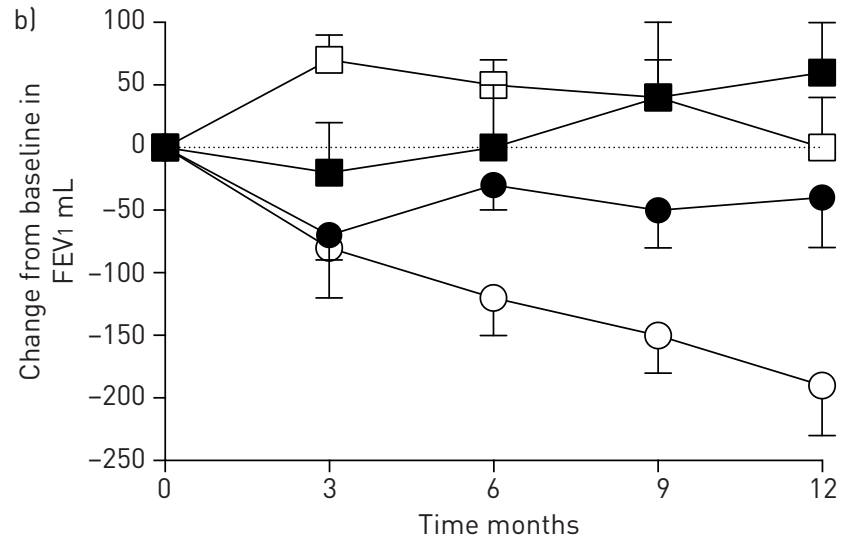

Placebo-pre-MP

Placebo-post-MP $\square$ Sirolimus-pre-MP

Sirolimus-post-MP

FIGURE 4 Mean \pm SE change from baseline to 12 months in forced expiratory volume in $1 \mathrm{~s}(\mathrm{FEV} 1)$ response in the MILES trial placebo and sirolimus groups, stratified by a) vascular endothelial growth factor (VEGF)-D levels and b) menopausal status. Pre-MP: pre-menopausal; post-MP: post-menopausal. a) Baseline VEGF-D $>600$ or $\leqslant 600 \mathrm{pg} \cdot \mathrm{mL}^{-1}$. Lung function in patients with baseline serum VEGF-D $\leqslant 600 \mathrm{pg} \cdot \mathrm{mL}^{-1}$ remained relatively stable throughout the 12-month monitoring period in both the placebo and sirolimus subgroups. Patients with serum VEGF-D $>600 \mathrm{pg} \cdot \mathrm{mL}^{-1}$ exhibited a faster decline in the placebo subgroup and improved treatment response to sirolimus. b) Pre-MP versus post-MP patients. Pre-MP patients on placebo had the highest rate of decline of lung function and had an overall stabilisation of FEV 1 decline on sirolimus. Post-MP patients on placebo remained relatively stable during the 12-month monitoring period and exhibited an overall improvement in their FEV1 following treatment with sirolimus.

Dose response and timing of adverse events

The mean \pm SD serum trough level of sirolimus in the treatment group was $7.2 \pm 3.4 \mathrm{ng} \cdot \mathrm{mL}^{-1}$. Sirolimus trough levels did not correlate with either FEV1 response or the incidence of adverse events. The majority of adverse events in both the sirolimus and placebo groups occurred in the time period immediately following drug initiation, and decreased over time (figure 6).

\section{Discussion}

The results of our analysis reveal that menopausal status has a significant effect on the natural history of disease progression in LAM, with pre-menopausal females exhibiting a faster rate of decline in FEV1 compared with post-menopausal females. Although a reduced rate of decline in FEV1 among post-menopausal females with LAM has previously been reported in retrospective cohort analyses of LAM patients $[3,6]$, the magnitude of difference before and after the menopause transition was much greater in

FIGURE 5 Percentage change from baseline in serum vascular endothelial growth factor (VEGF)-D levels and forced expiratory volume in $1 \mathrm{~s}$ (FEV 1 ) over 12 months. The placebo group exhibited progressive decline in FEV 1 with relatively stable VEGF-D levels, whereas the sirolimus group tended to have stable $\mathrm{FEV}_{1}$ with declining serum VEGF-D values during the 12-month treatment period.

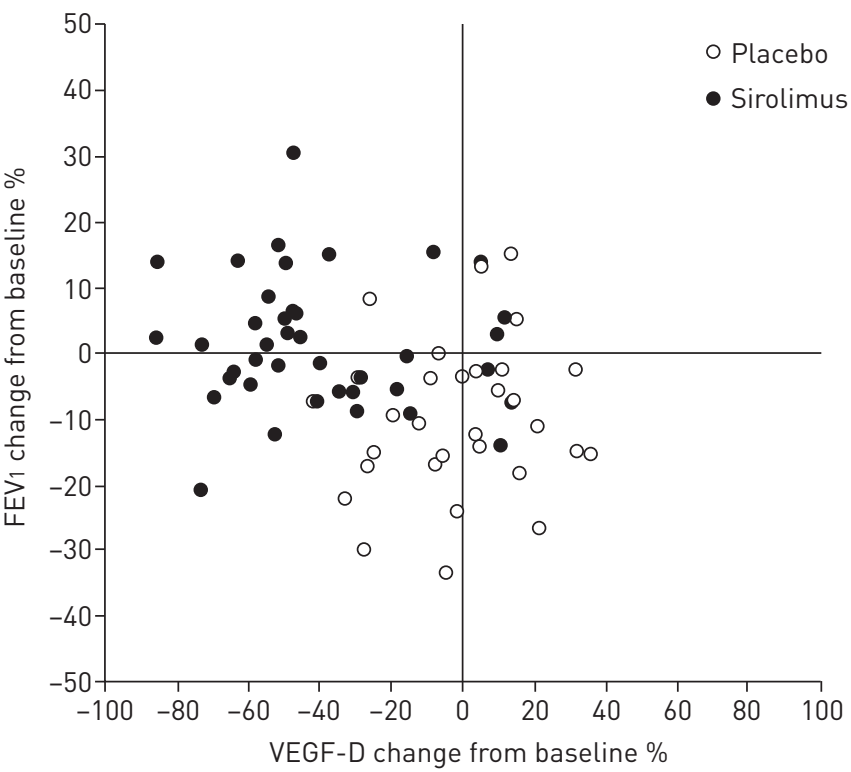


FIGURE 6 Time course of adverse events during the first 12 months of the MILES trial. The total numbers of adverse events are shown for both the sirolimus and placebo arms. In general, there were more adverse events in the sirolimus arm compared with the placebo arm and there was a decrease in the frequency of adverse events over the course of the trial in both arms.



this prospective analysis $(\sim 5$-fold compared with $<2$-fold). These data lend further credence to the role of sex steroids in the pathogenesis of LAM and suggest that menopausal status should be taken into consideration when making management decisions for LAM patients.

Despite vastly different rates of decline of FEV1 among pre- and post-menopausal LAM patients, both subgroups exhibited a beneficial response to treatment with sirolimus, although they varied by degree (figure $4 \mathrm{~b}$ ). The pre-menopausal subgroup had stabilisation of lung function decline after treatment with sirolimus, whereas the post-menopausal group had a trend towards improvement. Interestingly, post-menopausal patients treated with sirolimus also exhibited improvement in their rate of decline of DLCO, an effect that was not seen in either the overall MILES cohort or any of the other subgroups analysed. The menopausal status-based differential response to treatment with sirolimus remains unexplained and, because of the small numbers, will require validation in future studies. If confirmed, the data could support consideration of treatment of post-menopausal females with the goal of improvement (rather than merely stabilisation) of lung function.

In this study, race (Caucasian versus Asian) did not have an effect on the rate of decline of FEV1 (placebo arm) or the treatment response (sirolimus arm). Previous cohort analyses conducted on Asian and Caucasian subjects with LAM have yielded varying rates of decline of FEV1, suggesting an effect of race on the natural history of disease progression. For example, a recent analysis of 89 LAM patients in Japan enrolled in the Japanese National Research Project revealed a rate of decline in FEV1 of $47 \mathrm{~mL} \cdot \mathrm{year}^{-1}$ compared with $75 \mathrm{~mL} \cdot \mathrm{year}^{-1}$ in 275 LAM patients enrolled in the National Heart, Lung, and Blood Institute (NHLBI) intramural programme [3,7]. While racial and environmental differences may certainly play a role in the differences in these registry-based studies, the results from our prospective analysis demonstrating similar rates of decline of FEV1 and magnitudes of treatment response in Asian and Caucasian patients suggest that ascertainment bias and varying baseline disease severity are likely explanations for the race-associated discrepancies in FEV1 decline estimates between the MILES cohort and previously reported retrospective cohorts.

Race as well as menopausal status had an impact on the frequency and subtype of adverse events encountered in the placebo and sirolimus groups of the MILES trial. Understanding subgroup-dependent susceptibility to sirolimus-associated adverse events is useful for making treatment decisions and warrants further investigation in longitudinal, prospective cohorts. We have also shown that the incidence of adverse events is highest in the initial 3 months of sirolimus treatment. Declining rates of sirolimus-related adverse events over time on treatment have also been shown by other recent reports $[21,22]$. Collectively, these results highlight the need for close monitoring of LAM patients at the beginning of sirolimus treatment.

We have also found that TSC versus S-LAM did not impact the rate of decline of FEV1 or treatment response to sirolimus. It has been suggested that patients with TSC-LAM have milder and less progressive disease compared with patients with S-LAM $[9,10]$. However, many investigators believe that lead-time bias may play a role in this conclusion, in that patients with TSC-LAM are often discovered earlier through screening. A recent analysis from the NHLBI intramural programme has shown that the rate of decline of FEV1 is similar in TSC-LAM and S-LAM patients matched for baseline disease severity [23]. In addition, we have recently reported that LAM is the second most common cause of death in TSC and the most common cause of death in adult females with TSC [24]. Although the number of patients with TSC-LAM was small in the MILES trial, data presented here and recent reports from our group and others 
indicate that LAM can be as significant a healthcare burden and mortality risk for patients with TSC as it is for S-LAM patients, and requires close monitoring and appropriately aggressive and well-timed treatment interventions.

The presence of bronchodilator responsiveness on spirometry has been associated with a faster rate of decline in FEV1 [8, 25], as well as increased risk of progression to death or lung transplantation [5]. These results were not replicated in the MILES cohort, in that both subgroups exhibited a similar rate of decline of FEV1 as well as treatment response to sirolimus. The exact reason for these divergent results remains unknown, but they may be partially explained by the differences between cohorts in disease severity based on baseline FEV1. For instance, the baseline FEV1 was similar in the patients with or without bronchodilator responsiveness in the MILES cohort, but the baseline FEV1 was significantly lower in the patients with a positive bronchodilator response compared with the patients without a bronchodilator response in the previous analyses $[5,25]$.

Baseline lung function at the time of trial enrolment did not have an impact on either the rate of decline in FEV1 (in the placebo group) or on treatment response (in the sirolimus group). These results are in keeping with a recent analysis of the NHLBI LAM Registry which showed that the rate of decline in FEV1 is remarkably consistent across all categories after dividing patients on the basis of initial FEV1 [5]. Our results also highlight that treatment with sirolimus is beneficial even in patients with severe disease and that a trial of sirolimus treatment is warranted even in the most advanced cases of LAM.

The identification of surrogate biomarkers that are associated with meaningful outcomes (e.g. lung function decline or survival) in LAM is an unmet need that would greatly accelerate trials and reduce the numbers of patients required for interventional studies. Serum VEGF-D is a diagnostic biomarker for LAM and is recommended for use in the diagnostic algorithm prior to undertaking invasive diagnostic procedures in patients with suspected LAM [26]. In a post hoc analysis of the MILES trial, serum VEGF-D was also shown to have potential as a predictive biomarker of treatment response [13]. In this study, we found that a baseline serum VEGF-D cut-off level of $600 \mathrm{pg} \cdot \mathrm{mL}^{-1}$ identified a subset of patients that was more likely to progress and more likely to respond to treatment. The choice of $600 \mathrm{pg} \cdot \mathrm{mL}^{-1}$ as a cut-off was based on our previous report that this value represented the lower end of the range of VEGF-D levels that exhibited excellent diagnostic sensitivity and specificity for LAM among females with cystic lung disease [12]. The rate of decline in FEV1 in the high VEGF-D $\left(>600 \mathrm{pg} \cdot \mathrm{mL}^{-1}\right)$ group appeared to be fastest in the first 3 months of the study compared with the remainder of the first year (figure $4 \mathrm{a}$ ). This divergence in the rate of decline across the study duration is likely driven by selective dropout of the most severe patients from the placebo group.

Although not conclusive by themselves, post hoc analyses such as the current study are important for formulating future research questions and priorities, as well as informing the design of clinical trials. For example, if the differential impact of menopausal status and serum VEGF-D levels had been clearly established prior to MILES, and recruitment had been restricted to pre-menopausal patients with an elevated serum VEGF-D $>600 \mathrm{pg} \cdot \mathrm{mL}^{-1}$, the trial could have been powered with a sample size that was a fraction of the original enrolment target. Although one could argue that cohort stratification in this manner can limit the generalisability of results to the entire LAM population, in rare diseases with limited trial populations and finite resources this strategy of targeted recruitment of patients with the greatest potential for decline (based on clinical characteristics and biomarkers) could yield significant reductions in cost, time and risk exposure, and allow earlier access to treatment.

Strengths of this analysis included that the patients were enrolled prospectively and were randomised in a 1:1 ratio to placebo versus sirolimus in a double-blind fashion. This design allows us to compare the various subgroups not only in terms of the natural history of disease progression (i.e. in the placebo arm), but also assess treatment response. The various study-related parameters such as PFTs were collected at pre-specified intervals in a rigorous manner with real-time quality control and feedback. Serum VEGF-D measurements were performed in a CAP/CLIA-approved laboratory by personnel who were blinded to the clinical information. The major limitations of our analysis include the relatively low number of subjects in each of the subgroups and the post hoc approach. The randomised, placebo-controlled design likely introduced a selection bias leading to preferential recruitment of patients with more advanced and progressive disease, thus limiting the generalisability of these results to the entire LAM population. Lastly, these results represent observations from the first year of the MILES trial and may not be representative of long-term outcomes, either in terms of natural history of disease progression or treatment response with sirolimus.

\section{Conclusions}

In LAM patients with moderately severe disease enrolled in the MILES trial, the rate of decline in FEV1 in patients on placebo and the stabilising effect of sirolimus on FEV1 were similar after stratifying patients on 
the basis of Asian versus Caucasian race, TSC versus S-LAM, baseline FEV1 and the presence or absence of bronchodilator responsiveness on spirometry. Serum VEGF-D is a clinically useful diagnostic, prognostic and predictive biomarker for LAM. Menopausal status had a significant effect on the rate of decline of FEV1 in the placebo group; however, both pre- and post-menopausal females with LAM benefitted from treatment with sirolimus.

Author contributions: N. Gupta, H-S. Lee and F.X. McCormack had access to all of the data in the study and take full responsibility for the integrity of the data and the accuracy of the data analysis. H-S. Lee and J.P. Krischer performed the data analysis. All other listed authors contributed substantially in data acquisition and editing the manuscript. N. Gupta and F.X. McCormack had final responsibility for the decision to submit for publication.

Conflict of interest: N. Gupta has nothing to disclose. H-S. Lee has nothing to disclose. L.R. Young reports advisory board work for Boehringer Ingelheim and royalties for authorship from UpToDate, outside the submitted work; and has a patent Serum VEGF-D, no royalties issued. C. Strange reports grants for studies of LAM from Novartis, outside the submitted work. J. Moss has nothing to disclose. L.G. Singer has nothing to disclose. K. Nakata has nothing to disclose. A.F. Barker has nothing to disclose. J.T. Chapman has nothing to disclose. M.L. Brantly has nothing to disclose. J.M. Stocks has nothing to disclose. K.K. Brown reports grants from NHLBI, personal fees from AstraZeneca, Biogen, Galecto, MedImmune, Novartis, ProMetic, Patara, Third Pole, Galapagos, Boehringer Ingelheim, Theravance and Three Lakes Partners, conversation under CDA only from Genoa, other (submitted grant) from Roche/Genentech, outside the submitted work. J.P. Lynch has nothing to disclose. H.J. Goldberg has nothing to disclose. G.P. Downey has nothing to disclose. A.M. Taveira-DaSilva has nothing to disclose. J.P. Krischer has nothing to disclose. K. Setchell has nothing to disclose. B.C. Trapnell has nothing to disclose. Y. Inoue reports grants from Japanese Ministry of Health, Labor, and Welfare, during the conduct of the study. F.X. McCormack has a patent on serum VEGF-D testing. All royalties are waived to the parent institution, the University of Cincinnati.

Support statement: The study was supported by grants from the National Institutes of Health Office of Rare Disease Research, administered by the National Center for Research Resources (RR019498, to B.C. Trapnell and F.X. McCormack; RR019259, to H-S. Lee and J.P. Krischer), the Food and Drug Administration (FD003362, to F.X. McCormack), Canadian Institutes of Health Research (to G.P. Downey and L.G. Singer), National Institutes of Health (HL132950 to G.P. Downey), Pfizer Pharmaceuticals (to F.X. McCormack), the Japanese Ministry of Health, Labor, and Welfare (H 19 Rinshoshiken 008, to K. Nakata and Y. Inoue), the LAM Foundation (to F.X. McCormack), the Tuberous Sclerosis Alliance (Rothberg Courage Award, to F.X. McCormack), Cincinnati Children's Hospital Medical Center (Institutional Clinical and Translational Science Award 1UL1RR026314-01, to L.R. Young and F.X. McCormack; Translational Research Initiative Award, to F.X. McCormack and B.C. Trapnell), Vi and John Adler, and the Adler Foundation. J. Moss and A.M. Taveira-DaSilva were supported by the Division of Intramural Research, National Institutes of Health, National Heart, Lung, and Blood Institute. Pfizer provided the study drug and money for study visit costs. Pfizer had no role in the study design; in the collection, analysis and interpretation of data; in the writing of the report; or in the decision to submit the paper for publication. Funding information for this article has been deposited with the Crossref Funder Registry.

\section{References}

1 Gupta N, Vassallo R, Wikenheiser-Brokamp KA, et al. Diffuse cystic lung disease. Part I. Am J Respir Crit Care Med 2015; 191: 1354-1366.

2 Henske EP, McCormack FX. Lymphangioleiomyomatosis - a wolf in sheep's clothing. J Clin Invest 2012; 122: 3807-3816.

3 Taveira-DaSilva AM, Stylianou MP, Hedin CJ, et al. Decline in lung function in patients with lymphangioleiomyomatosis treated with or without progesterone. Chest 2004; 126: 1867-1874.

4 McCormack FX, Inoue Y, Moss J, et al. Efficacy and safety of sirolimus in lymphangioleiomyomatosis. $N$ Engl J Med 2011; 364: 1595-1606.

5 Gupta N, Lee HS, Ryu JH, et al. The NHLBI LAM Registry: prognostic physiological and radiological biomarkers emerge from a 15-year prospective longitudinal analysis. Chest 2019; 155: 288-296.

6 Johnson SR, Tattersfield AE. Decline in lung function in lymphangioleiomyomatosis: relation to menopause and progesterone treatment. Am J Respir Crit Care Med 1999; 160: 628-633.

7 Hayashida $M$, Yasuo $M$, Hanaoka $M$, et al. Reductions in pulmonary function detected in patients with lymphangioleiomyomatosis: an analysis of the Japanese National Research Project on Intractable Diseases database. Respir Investig 2016; 54: 193-200.

8 Taveira-DaSilva AM, Hedin C, Stylianou MP, et al. Reversible airflow obstruction, proliferation of abnormal smooth muscle cells, and impairment of gas exchange as predictors of outcome in lymphangioleiomyomatosis. Am J Respir Crit Care Med 2001; 164: 1072-1076.

9 Avila NA, Dwyer AJ, Rabel A, et al. Sporadic lymphangioleiomyomatosis and tuberous sclerosis complex with lymphangioleiomyomatosis: comparison of CT features. Radiology 2007; 242: 277-285.

10 Taveira-DaSilva AM, Pacheco-Rodriguez G, Moss J. The natural history of lymphangioleiomyomatosis: markers of severity, rate of progression and prognosis. Lymphat Res Biol 2010; 8: 9-19.

11 Young LR, Inoue Y, McCormack FX. Diagnostic potential of serum VEGF-D for lymphangioleiomyomatosis. $N$ Engl J Med 2008; 358: 199-200.

12 Young LR, Vandyke R, Gulleman PM, et al. Serum vascular endothelial growth factor-D prospectively distinguishes lymphangioleiomyomatosis from other diseases. Chest 2010; 138: 674-681.

13 Young L, Lee HS, Inoue Y, et al. Serum VEGF-D concentration as a biomarker of lymphangioleiomyomatosis severity and treatment response: a prospective analysis of the Multicenter International Lymphangioleiomyomatosis Efficacy of Sirolimus (MILES) trial. Lancet Respir Med 2013; 1: 445-452. 
14 Inoue Y, McCormack FX, Lee HS, et al. The MILES trial: the effect of Asian race on outcomes in patients with lymphangioleiomyomatosis. Am J Respir Crit Care Med 2012; 185: A4444.

15 McCormack FX, Inoue Y, Moss J, et al. The MILES trial: effect of menopausal status on disease progression and treatment responses in lymphangioleiomyomatosis. Am J Respir Crit Care Med 2012; 185: A4442.

16 Miller MR, Hankinson J, Brusasco V, et al. Standardisation of spirometry. Eur Respir J 2005; 26: 319-338.

17 Wanger J, Clausen JL, Coates A, et al. Standardisation of the measurement of lung volumes. Eur Respir J 2005; 26: 511-522.

18 Macintyre N, Crapo RO, Viegi G, et al. Standardisation of the single-breath determination of carbon monoxide uptake in the lung. Eur Respir J 2005; 26: 720-735.

19 Pellegrino R, Viegi G, Brusasco V, et al. Interpretative strategies for lung function tests. Eur Respir J 2005; 26 : 948-968.

20 McNeish D. Small sample methods for multilevel modeling: a colloquial elucidation of REML and the KenwardRoger correction. Multivariate Behav Res 2017; 52: 661-670.

21 Takada T, Mikami A, Kitamura N, et al. Efficacy and safety of long-term sirolimus therapy for Asian patients with lymphangioleiomyomatosis. Ann Am Thorac Soc 2016; 13: 1912-1922.

22 Bee J, Fuller S, Miller S, et al. Lung function response and side effects to rapamycin for lymphangioleiomyomatosis: a prospective national cohort study. Thorax 2018; 73: 369-375.

23 Taveira-DaSilva AM, Jones AM, Julien-Williams P, et al. Severity and outcome of cystic lung disease in women with tuberous sclerosis complex. Eur Respir J 2015; 45: 171-180.

24 Zak S, Mokhallati N, Su W, et al. Lymphangioleiomyomatosis mortality in patients with tuberous sclerosis complex. Ann Am Thorac Soc 2018; in press [https://doi.org/10.1513/AnnalsATS.201807-471RL].

25 Taveira-DaSilva AM, Steagall WK, Rabel A, et al. Reversible airflow obstruction in lymphangioleiomyomatosis. Chest 2009; 136: 1596-1603.

26 McCormack FX, Gupta N, Finlay GR, et al. Official American Thoracic Society/Japanese Respiratory Society Clinical Practice Guidelines: Lymphangioleiomyomatosis Diagnosis and Management. Am J Respir Crit Care Med 2016; 194: 748-761. 